\title{
LA MATEMÁTICA EN LA METACOGNICIÓN Ó LA METACOGNICIÓN EN LA MATEMÁTICA: METACOGNICIÓN - COMPLEJIDAD - MATEMÁTICA
}

\author{
MATHEMATICS IN METACOGNITION OR METACOGNITION IN \\ MATHEMATICS: METACOGNITION - COMPLEXITY - MATHEMATICS
}

Milagros Elena Rodríguez ${ }^{1}$

\begin{abstract}
Resumen: Urge el pensar profundo en estadios metacognitivos de alto impacto en el ser humano; es una de las distinciones de otros habitantes de la tierra. La lógica dialéctica y su execrar de la enseñanza develan una intencionalidad colonial. Con el transmétodo la deconstrucción, en una decolonialidad planetaria, se analizan emergente re-ligantes de la triada: metacognición-complejidad-matemática. Ubicada en la línea de investigación titulada: Educación Matemática Decolonial Transcompleja. Como conclusiones los procesos mentales: concreto-abstractos, análisis-síntesis, inducción-deducción, verdad-error, absolutorelativo, teoría-práctica, macro-micro; entre otros; se declaran inseparables. Re-ligando emerge el gran espectro que pensar profundo es hacer matemática es tener un pensamiento complejo. Las estrategias complejas como emergentes re-ligantes develadoras de un pensar profundo en la enseñanza, son primerísima actividad de dicha ciencia. La matemática como ejercicio no soslayador decolonial lleva a la metacognición y esta no se desune de la matemática. La tríada: metacognición - complejidad - matemática no se desune. Es de urgente consideración.
\end{abstract}

Palabras clave: Enseñanza de la matemática; Re-ligar; Metacognición; Complejidad; Decolonialidad.

Abstract: It is urgent to think deeply in metacognitive stages of high impact in the human being; it is one of the distinctions of other inhabitants of the earth. The dialectical logic and its execration of teaching reveal a colonial intention. With the deconstruction transmethod, in a planetary decoloniality, emergent re-binders of the triad are analyzed: metacognition-complexity-mathematics. Located in the research line entitled: Transcomplex Decolonial Mathematical Education. As conclusions the mental processes: concreteabstract, analysis-synthesis, induction-deduction, truth-error, absolute-relative, theory-practice,macromicro; among others; they are declared inseparable. Re-linking the great spectrum emerges that deep thinking is doing mathematics is having complex thinking. Complex strategies as emergent re-binding revealing of a deep thinking in teaching, are the very first activity of this science. Mathematics asa decolonial non-circumventing exercise leads to metacognition and this does not get rid of mathematics. The triad: metacognition - complexity - mathematics does not fall apart. It is of urgent consideration.

Keywords: Mathematics teaching, Re-linking, Metacognition, Complexity, Decoloniality.

"La matemática toca al hombre profundamente y, como la educación, tiene por función revelar el hombre a sí mismo. El que no posee una Educación Matemática se halla privado de algo que es esencial al hombre". George Papy (1980).

"El re-ligar es una práctica emergente del pensamiento filosófico transmoderno”. Milagros Elena Rodríguez (2019a).

\footnotetext{
${ }^{1}$ Postdoctora en Ciencias de la Educación por la Universidad Nacional Experimental de las Fuerzas Armadas (UNEFA), Doctora en Patrimonio Cultural por la Universidad Latinoamericana y el Caribe (ULAC), Doctora en Innovaciones Educativas por la Universidad Nacional Experimental de las Fuerzas Armadas (UNEFA), Licenciada y Magister Sienciaturum en Matemáticas por la Universidad de Oriente (UDO), Cumaná, Sucre Venezuela. E-mail melenamate@ hotmail.com
} 


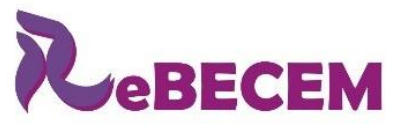

DOI: https://doi.org/10.33238/ReBECEM.2020.v.4.n.4.24986

\section{Rizoma introito: La metacognición colonial: aperturas de la complejidad a la}

tríada: metacognición - complejidad - matemática

Partimos de la premisa de los epígrafes que presentan esta investigación; las matemáticas tocan estructuras mentales profundas en el ser humano; quien no domina la esencia de la matemática, sus primeras conceptualizaciones, está impedido para tomar decisiones en su cotidianidad; y más para realizar estudios de niveles universitarios que les llevan a encontrar fracasos escolares bien conocido en la educación.

La educación ha sido objeto de colonización; queremos mostrar nuestra intencionalidad en presente discurso; "debemos desoccidentalizar las ciencias de la educación y develar la doctrinalidad de la educación y la formación, el proceso que oculta la pedagogía en su propuesta de la noción de formación, así como la contradicción entre formación y emancipación. La doctrinalidad es la cara oculta de la formación" (ORTIZ; ARIAS; PEDROZO, 2018, p.1).

Ese accionar colonial lleva a esencias del proyecto transmoderno: la transmodernidad es una perspectiva teórica, metodológica y ética (DUSSEL, 2005) que tiene la misión e intencionalidad de romper con la colonialidad del poder, del saber y del ser de Occidente. Siendo la postmodernidad parte del proyecto modernista, entonces modernidad y postmodernidad tienen por proyecto la colonialidad.

Esta investigación es antimétodo; va más allá de los métodos coloniales; se dedica todo un rizoma, más adelante se explicita su significancia, al transmétodo la deconstrucción como transmétodo transmodernista; que es desde luego esencia de la indagación para emerger con ella categorizaciones y ejercicios metacognitivos de alto nivel en el cumplimiento del objetivo complejo, que prontamente conceptualizamos. El sujeto investigador así tiene una inmersión directa en la indagación; contrariamente cuando en la tradicionalidad el sujeto investigador permanece fuera de la investigación. Acá la autora de doliente y agente de cambio de la problemática. Se pide al lector una apertura mental, decolonial, en la lectura de la investigación.

Por otro lado, en la crisis de la modernidad-postmodernidad-colonialidad, el pensar libre elevado abstracto ejemplificado a hacer el bien, en tanto tenemos la capacidad desarrollado la solidaridad para poder colaborar en la realización de la humanidad, es una problemática no atendida; no conveniente a su proyecto soslayador. 

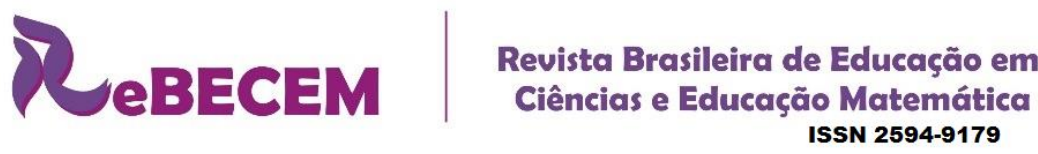

DOI: https://doi.org/10.33238/ReBECEM.2020.v.4.n.4.24986

Es observable en diversos campos laborales, y en los cotidianos la incapacidad mostrada al presentar aún en profesionales la impulsividad, falta de precisión y exactitud en la recopilación de datos cotidianos de su medio de trabajo, definición imprecisa de un problema que se presente, falta de la necesidad de evidencia lógica ante un pensamiento mecánico, digo impreciso que se muestra en el comunicar, y con ello la falta de precisión al comunicar las respuestas a una determinada contingencia.

Se consigue carencias en el pensamiento lógico, y en la lógica del pensamiento de muchas personas que no han desarrollado su capacidad de pensar por el mero disfrute de desarrollar habilidades para desenvolverse. Se ha formado profesionales para sólo dar respuestas a lo que conviene en los medios de producción. No ha convenido, en muchos casos el pensar para desenvolverse como ciudadanos exitosos en tanto resolver problemas para la vida, la alta demanda existente lo muestra. Son resultados de los estudios de la decolonialidad del saber, ser y hacer en la educación. El ejercicio de poder en la enseñanza “como soslayación en las aspiraciones a educarse y llegar a ascender y construir cada día, re-construir sus teorías desde aplicabilidades nuestras, desde la cotidianidad y saberes soterrados desde el Sur" (RODRÍGUEZ, 2020a, p.127). Y de otras latitudes, conocimientos que deben salvaguardarse.

En época de pandemia, pleno año 2020, vemos como es de obligatoriedad el manejo de las tecnologías para comunicarse, sin embargo nos conseguimos con analfabetas tecnológicos, personas sin las competencias mínimas para manejar computadoras y celulares de alta generación, siendo profesionales universitarios, con miedo a re-aprender, con predisposiciones que le impiden el pensar y resolver en contingencia adecuadamente. No es casualidad, que en la mayoría de los casos, que sean analfabetas matemáticos (RODRÍGUEZ, 2020b), ¿Qué relación tienen estas condiciones? ¿Las matemáticas permiten el desarrollo del pensamiento?

Iremos perfilando la respuesta a tan inquietante pregunta, "las matemáticas permiten el desarrollo de una lógica de pensamiento, o de un pensamiento lógico" (PEÑALVA ROSALES, 2010, p.135), pero: ¿qué ha pasado con el aprendizaje de la matemática en las universidades que no ha calado en ese desarrollo del pensamiento lógico, en muchos profesionales? Se ha usado la ciencia mecánicamente con pasos algorítmicos para resolver problemas sin la disertación, sino la memorización mecánica sin la posibilidad de discernir discente-docente de ese pensar lógico, entendiendo y desarrollando niveles de abstracción ascendente del pensamiento. 


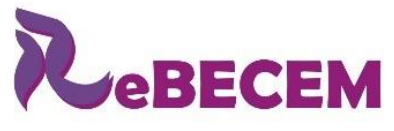

DOI: https://doi.org/10.33238/ReBECEM.2020.v.4.n.4.24986
Revista Brasileira de Educação em

Ciências e Educação Matemática

El estudio de como aprender y como el cerebro codifica se emociona, se motiva para aprender no es motivo de estudio en la docencia clásica de la modernidadpostmodernidad-colonialidad. El disfrute del pensar como mero hecho; comparado por ejemplo con el deportista, el futbolista que llega casi al máximo desarrollo de su cuerpo en el entrenamiento es motivo central para ser un exitoso deportista; pero, en el arte de desarrollar el pensar, el cerebro activo es escaso en muchas ocasiones el ejercitarse; ¿es común ver estos ejercicios de conversaciones profundas deleitándose para aprender a pensar?

Por ejemplo, con el uso de las tecnologías, actualmente en época de pandemia, vemos como algunos profesionales se dedican a copiar y pegar violando el derecho de autor, sin discernimiento, primero el moral, ético del respeto a la autoría y la debida cita; segundo el uso de esas ideas para elevar metacognitivamente su pensar, para ir a buscar ideas más innovadoras, creativas y profundas de las que se han consultado. Preocupa, ¿Cómo los niños investigan en las redes tecnológicas? ¿Siguen trasmitiendo el antivalor de copiar y pegar, y propagando los errores epistemológicos que se comunican en las redes, tal como lo hacen muchos profesionales de todos los niveles? Los errores epistemológicos es la transmisión de las malas conceptualizaciones que llevan conocimientos de la matemática equivocados.

Sin discernir en la respuesta, en el pensar de las consecuencias hay una gravedad que se ha venido propagando con la globalización, el ser imitadores; y la metacognición se queda en la repetición de un nivel ínfimo que sólo lleva a saber copiar y pegar; si la metacognición no llega a desarrollarse adecuadamente cuando el ser humano no discierne actua como máquina repitiendo para memorizar (MORÍN, 1999); son resultados que se plasman en obra: La Cabeza Bien Puesta: Repensar la reforma. Reformar el pensamiento, del mencionado autor. Eso, en muchas ocasiones hace que la formación de profesionales no sea en el desarrollo del pensamiento crítico, lógico de alto nivel con la interpretación, y discernimiento entre los errores; pues no hay una provocación del pensar profundo. En ello, vamos direccionado en la indagación.

Por otro lado, si la matemática es por excelencia en el desarrollo de la metacognición, se pregunta, en vista que la autora es sujeto participe de la investigación por la liberación que se ha explicitado del sujeto investigador por el transmétodo, experiencia en la formación y como matemático y en años de servicio en la Educación Universitaria pregunta; en muchas oportunidades: ¿Por qué en las universidades donde se ha enseñado matemáticas a profesionales de diversas disciplinas no se ha tenido el 
DOI: https://doi.org/10.33238/ReBECEM.2020.v.4.n.4.24986

éxito necesario en el pensar profundo? Es porque entre otras líneas constitutivas de la problemática, se ha usado:

La lógica formal, como tradicionalmente la conocemos, donde el cumplimiento de formas y reglas para dar validez a las conclusiones es irrestricto, los caminos construidos mediante las matemáticas pueden volverse camisas de fuerza para el desarrollo libre del pensamiento y de la capacidad de aprender a aprender (PEÑALVA ROSALES, 2010, p.137).

Y con esa lógica formal desconocida hasta por los mismos docentes impuestas con el único propósito de memorizar mecánicamente y no de pensar para discerní lo que no está bien en los mismos pasos necesarios de la formalidad, que no se le niega a la ciencia matemática; pero que lo irrestricto sin el pensar profundo ha matado la belleza de la matemática ante las personas. He allí, una de las provocaciones que las creencias y afectividad negativa se han llevado a su máximo nivel en la enseñanza de la matemática.

Donde queda entonces para terminar en esta parte con la discusión de la lógica formal, ¿dónde queda la enseñanza de la lógica dialéctica en las matemáticas desde los primeros niveles de educación? La dialéctica porque con ella, como en los diálogos socráticos a los que Platón ha dejado a la humanidad se deleitaba el pensar profundo como el centro propósito del dialogo y no la búsqueda de respuesta ante el tema del dialogo únicamente (PLATÓN, 2012).

La lógica dialéctica lleva a la ecología de los saberes entre procesos del pensamiento que parecen disimiles, pero que se abrazan en el pensar como lo: concretoabstracto, análisis-síntesis, inducción-deducción; entre otros. Por ello en la modernidadpostmodernidad se consiguen escasos pensares profundos donde esos diálogos den, la cuestión de la abstracción de "las determinaciones; el ascenso dialéctico de lo abstracto a lo concreto; la construcción sintética del todo concreto; la problemática en tomo a las categorías" (DUSSEL, 1985, p.48) que en investigadores tradicionalistas se encuentra la escasez del pensar profundo.

El otro problema del porque la matemática no ha calado en el pensar profundo, es la intencionalidad de la enseñanza modernista-postmodernista-colonial de privar a la enseñanza y construcción de sus teorías de la historia y su filosofía que la ha provisto desnuda en la vida de los ciudadanos, lo que ha hecho que se presente con desinterés y cobre el espacio de la matemática, un pensamiento abismal con la vida del discente y su cotidianidad.

Nos preguntamos: ¿a qué elite le conviene ligar lo estricto incambiable de cómo se presentan por ejemplo, los pasos del cálculo con la obediencia sumisa si discernir? 


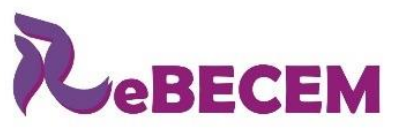

Revista Brasileira de Educação em

Ciências e Educação Matemática

DOI: https://doi.org/10.33238/ReBECEM.2020.v.4.n.4.24986

¿Se ha usado la matemática en el aula para ello?, la Educación Matemática ha venido siendo "capaz de operar como un arma secreta del imperialismo occidental" (SKOVSMOSE, 2012, p.270)

De la misma manera respondiendo la pregunta anterior; el gran matemático Georges Léopold Anatole Papy matemático belga quien en este lado del mundo en Argentina y Uruguay tuvo su marca en la enseñanza, afirma que "aquellos que piensan que el hombre tiene que obedecer y ejecutar pueden preferir al individuo que sabe mecánicamente las reglas de cálculo" (PÉREZ, 1980, p.43). La matemática, está ligada a los procesos del pensamiento, del sentir, de la emoción y del espíritu; porque el ser humano siempre tiene que demostrar, que poner en evidencia conceptos, la necesidad de darse una forma interna de explicación, nótese la obligatoriedad de una matemática esplendida por excelencia en la vida.

Antiguamente, en la enseñanza primera de la matemática cuando se cuestionaba la enseña del profesor de matemáticas era como poner en duda la matemática misma; no hay duda que el método del docente y el objeto matemático estaba profundamente ligados. Se avecina respuestas al rechazo a la matemática, no entiendo el método que me impone el docente, rechazo la matemática.

Un eje constitutivo de la crisis, es el distanciamiento de la matemática en la vida en lo concreto en la cotidianidad y cultura, e ir de lo concreto a lo abstracto; el niño tiene un pensar concreto en su niñez con sus juegos; al enseñarle matemática se le impone el pensamiento abstracto y como el docente no conoce la matemática del pensar profundo no va de lo concreto del niño a los abstracto del número (RODRÍGUEZ, 2010); en ese momento destruye la posibilidad de que se gane el discente para el estudio de la matemática.

Al fin, entre tantas razones la matemática por excelencia para el desarrollo del pensamiento profundo del ser humano se diluye de cumplir su propósito del desarrollo de pensamientos metacognitivos de alto nivel, en la predisposición o en la imposición inadecuada; en la mayoría de los casos. Es de saber que la coacción, predisposición, creencias negativas impide el pensar profundo. $\mathrm{Y}$ es bien sabido que el cerebro necesita emocionarse para aprender (FERNÁNDEZ, 2010).

Por otro lado, el desarrollo en la transmodernidad de las obras de Enrique Dussel se ha llevado en pensar abstracto-concreto, en las lecturas de Karl Marx en su obra: el capital el comentario de los Grundrisse, le ayudo a Enrique Dussel a "construir, como categorías analíticas estrictas, los conceptos de "pobre” y "pueblo" - uno el singular y otro 
DOI: https://doi.org/10.33238/ReBECEM.2020.v.4.n.4.24986

el colectivo histórico" (DUSSEL, 1985, p.18). Intento decir aquí que bajo el pensamiento decolonial, develando la verdadera esencia de la matemática, moverse en la arena de lo abstracto-concreto ha sido fructífero en estos investigadores decoloniales para que emerjan otras categorías vedadas de la modernidad-postmodernidad en ir a otros estadios del pensamiento profundo. Es decir ha sido un ejercicio metacognitivo de alto nivel en los propios investigadores decoloniales para poder develar la cara oculta de la soslayación modernista.

Por ello, acá la investigación se mueve en escenarios transmodernistas para ir a analizar en rizomas como: la matemática como aperturas y excelsitud de la metacognición, estrategias metacognitivas complejas, como emergentes re-ligantes en la enseñanza de la matemática y finalmente que desde allí emerja el objeto transdisciplinar: metacognición-complejidad-matemática.

Se explicará adecuadamente en los rizomas siguientes y además no se promete no regresar a la crisis de la enseñanza de la matemática en el discurso; para ver cómo se llegó a ser lo que es y cómo la metacognición es un proceso de colonialidad de las mentes, eje constitutivo de dominancia. Pues no ha convenido que los dominados lleguen a un pensar profundo que es metacognitivo, de la mente, pero también espiritual emocional constitutivo de la con-formación del ser: cuerpo-mente-alma-espíritu envuelto en la tierra-patria. Todo ello se devela en lo adelante en la investigación.

En el discurso que continúa se develan concepciones modernistas-postmodenistas de la metacognición y la cognición que enriquecerán el discurso acudiendo a escenarios complejos y transdisciplinarios para poder de-velar en las fronteras de las disciplinas no comunicables lo indecible que haría re-ligar (RODRÍGUEZ, 2019a) el pensamiento hacia escenarios profundos de un pensar complejo, que es deseable en estos tiempos donde los problemas complejos sobreabundan bajo miradas disyuntivas desgarradoras de la realidad. Por ello, en mi recorrido investigativo he deconstruido al pensar, y voy con Edgar Morín, La cabeza bien puesta. Repensar la reforma. Repensar el pensamiento (MORÍN, 1999), pues “vale más una cabeza bien puesta que una repleta” (MONTAIGNE, 2007, p.4).

La transdisciplinariedad, propone que no se fragmente la realidad en base a los vanidosos principios dialécticos de superioridad, (...) lo transdisciplinar se propone una actitud siempre dialógica, reconociendo la complejidad susceptible de encontrarse en todo nivel de análisis" (OLANO, 2019, p.12). La transdisciplinariedad confronta la desunión del conocimiento y va a buscar esencias de conjunción 


\section{Rizoma transmetodológico: la deconstrucción como transmétodo transmodernista}

Los transmétodos son esencias de la investigación transcompleja, la transcomplejidad es:

Transmetódica, concepto que envuelve lo metametódico; constituye y postula un ascenso en el pensamiento (...) se observa un rebasamiento de los tradicionales trasfondos paradigmáticos (...) es un ejercicio de reflexión donde se repiensa permanentemente el statu quo de las ciencias (ZAÁ, 2017, p.91).

Desde luego, dejan estas últimas de erigir como las eruditas del saber para abrirse a lo olvidado y desmitificado. La deconstrucción "como transmétodo sólo es posible en la transcomplejidad bajo el proyecto transmoderno" (RODRÍGUEZ, 2019b, p.13).

Se analizan los emergente re-ligantes de la tríada: metacognición-complejidadmatemática, como objetivo complejo de la investigación. Se ubica en la línea de investigación titulada: Educación Matemática Decolonial Transcompleja. La deconstrucción como transmétodo de investigación “es libre al máximo, anti-dogmática, no tiene ninguna transmetodología fija, su objetivo es debilitar el pensamiento filosófico occidental, destruir las concepciones colonizantes en todas sus formas y significados" (RODRÍGUEZ, 2019b, p.43). Así, este transmétodo, busca involucrarse en procesos develadores encubiertos en discursos, prácticas, acciones y discursos impresos, para con esto descomponerlos, desarticularlos y re-ligarlos con un sentido otro en la temporalidad que nos ocupa, desde una visión de hologramática, del todo y sus partes, de las partes y el todos en un mecanismo permanente de bucle recursivo, (MORÍN, 2005a), acto que es considerado por la autora como "proceso descolonizador" (RODRÍGUEZ, 2019b, p.54), que lleva a los sujetos que la utilizan a la creación de transepistemologías que invitan a la emergencia de movimientos emancipatorios; que van de la mano con la enseñanza de la matemática.

Es de clarificar que estos emergente religantes están cargados de subjetividades y de categorías originales, muy distantes de la tradicionalidad investigativa de la enseñanza de la matemática; tales como: amor, Dios nuestro creador: la autora ser humano cristiana no se desliga de sus creencias y afectividades en la construcción transmetódica, también categorías como afecto, pensamiento, opresión entre otras. Se invita al lector a su compresión en la línea de investigación titulada: Educación Matemática Decolonial 


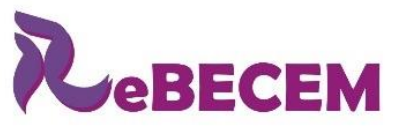

Revista Brasileira de Educação em

Ciências e Educação Matemática

DOI: https://doi.org/10.33238/ReBECEM.2020.v.4.n.4.24986

Transcompleja que tiene varios resultados en: Rodríguez (2020a), Rodríguez (2020b), Rodríguez (2020c) y Rodríguez (2020d). Ellas aportan sustantivamente a la presente indagación.

Se hace "desenmascaramiento del pensamiento occidental, de la modernidad, los ideales tradicionales impuestos de la educación y sus representaciones sociales" (RODRÍGUEZ, 2019b, p.7) en tanto la enseñanza de la matemática, la metacognición, cognición son objetos de poder opresivo de la colonialidad.

Con la deconstrucción la autora estará en un "ir y venir que va a la criticidad; pero también a la reconstrucción" (RODRÍGUEZ, 2019b, p.9) de la metacognición y de algunas estrategias para la enseñanza de la matemática para ir a estadios metacognitivos y cognitivos complejos en el ser humano. Así las cosas, la autora va a una actitud creadora y transvisionaria. Se resalta a los lectores que los transmétodos liberan al sujeto investigador; asi sus subjetividades y experiencias, creencia y otras actitudes no regularizables por la forma tradicional de investigación están presenten es toda la indagación. Cuestión que no ocurre con las investigaciones modernistas-postmodernistascoloniales.

Los emergente, re-ligantes de la tríada: metacognición-complejidad-matemática son posibilidades en lo complejo y transdisciplinar, con la deconstrucción, como transmétodo transcomplejo antidogmático, destruye las concepciones coloniales y no hace un barrido de las investigaciones de la metacognición, sino que apertura por ello nuevos escenarios para la metacognición decolonial.

Para ello, en los emergente re-ligantes de la tríada: metacognición-complejidadmatemática, la deconstrucción es re-constructiva y va conjugar y analizar en rizomas: la matemática como aperturas y excelsitud de la metacognición, estrategias metacognitivas complejas, como emergentes re-ligantes en la enseñanza de la matemática y finalmente que desde allí emerja el objeto transdisciplinar: metacognición-complejidad-matemática; para finalizar en conclusiones de apertura en un pensar profundo.

Se quiere hacer entrever que este es un tema del objeto transdisciplinar del orden de pensar profundo, que pude conllevar tomando esta investigación como inicio; hacia construcciones de tesis doctorales. Pero, que con la concepción del uso de rizomas se comienzan las mesetas; por ello la organización de esta indagación no responde a ningún modelo estructural o generativo; es un: 
DOI: https://doi.org/10.33238/ReBECEM.2020.v.4.n.4.24986

que se desarrolla evitando cualquier orientación hacia un punto culminante o hacia un fin exterior (DELUEZE; GUATTARI, 2004, p.26).

La palabra rizoma deviene de la Biología, la denotación en la indagación no es una casualidad, es la profunda transdisciplinariedad que no le atribuye apellidos consensuado dueños en las concepciones o nominaciones: la denominación de rizoma en la estructura de la investigación, "se trata de una anti-genealogía que rompe con las estructuras estáticas divisorias de presentar las indagaciones en las que las partes se dividen indisolublemente en un ir si un venir" (RODRÍGUEZ, 2019b, p.4). Ahora, aprendamos a pensar profundo en las mesetas en construcción, desde el develar de la dominación de la disyuntiva metacognición impuesta como objeto de poder para la colonialidad de las mentes. Intentamos construir bases sólidas a las mesetas en la deconstrucción como transmétodo, sólo posible en la decolonialidad planetaria, en la transmodernidad. Se quiere pensar profundo, ver en donde el pensar disyuntivo impidió la metacognición de alto nivel, George Papy lo afirma:

Las matemáticas nos vinculan con el Ser, con la realidad. (...) constato que las matemáticas tocan estructuras psicológicas profundas (...) podemos decir que el dominio del lenguaje matemático ejerce un efecto terapéutico (PÉREZ, 1980, p.45).

Se sigue entonces, "interpelando los territorios temáticos del conocimiento" (RODRÍGUEZ, 2019b, p.6) de matemática en la metacognición y la metacognición en la enseñanza de la matemática. De allí la conformación de la tríada: metacognicióncomplejidad-matemática. Que se solicita la lector la apertura mental a investigaciones más allá de la tradicionalidad soslayadora; nos inmersionamos seguidamente en los resultados.

\section{Rizoma: La matemática como aperturas decoloniales en la excelsitud de la metacognición}

La grandeza y profundidad de abstracción del conocimiento matemático es por excelencia el convocado a desarrollar niveles de excelsitud en los seres humanos. Esto es posible, primero bajo el develar complejo que re-liga las amarras impuestas de la matemática para la solución de problemas, como primera tarea de la ciencia legado de la humanidad; olvidándose del aporte del pensar profundo. Se señala que la matemática 


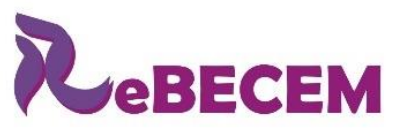

Revista Brasileira de Educação em

Ciências e Educação Matemática

DOI: https://doi.org/10.33238/ReBECEM.2020.v.4.n.4.24986

"aporta de manera clara y contundente al desarrollo de la metacognición, y por ende a la capacidad de aprender a aprender” (PEÑALVA ROSALES, 2010, p.135).

Por ello, en la consideración transdisciplinar de los conocimientos, la matemática debe ser incluida en todos los programas curriculares de las carreras universitarias, pero para desarrollar en primer lugar, el pensamiento profundo e ir a coadyuvar al estudio de los saberes; en el elucidar en un pensamiento profundo debemos ir, por ejemplo, con el gran matemático Godofredo Guillermo Leibnitz, quien invento el cálculo diferencial, dedicó los últimos años de su vida a la filosofía, en grandes obras de pensamiento profundo: La Monadología, La Teodicea y los Nuevos ensayos, en la primera expresa que:

Se fundan nuestros razonamientos en dos grandes principios: el de la contradicción, en virtud del cual juzgamos falso lo que ésta encierra, y verdadero lo que es opuesto a lo falso o contradictorio (LEIBNIZ, 1889, p.18).

Ahora, en el discurso vamos a ir a los conceptos que parecen contrapuestos y contradictorios, tales como concreto-abstracto, análisis-síntesis, inducción-deducción (PEÑALVA ROSALES, 2010) para ejemplificar como la matemática tendrá aperturas decoloniales a como primerísima en la metacognición.

Es ahora preciso, donde la autora re-ligar es necesaria continuación de la indagación; la metacognición bajo la metacomplejidad para ir a pensamientos profundos va en el ser humano a el discernimiento y el abrazo de "la coexistencia de lo opuesto, la incorporación del azar, la incertidumbre y la toma de conciencia de lo que conoces o aprendes en la toma de conciencia" (GONZÁLEZ, 2017, p.30) donde lo:

Metacomplejo aquello que no puede resumirse en una palabra maestra, en una síntesis, aquello que no puede retrotraerse a una ley, aquello que no puede reducirse una idea simple o mecanicista (GONZÁLEZ, 2017, p.29).

Para ello, lo complejo "no puede resumirse en el término complejidad" (MORÍN, 2005b, p.9), ni en la simple metacognición reduccionista. En esa aceptación de la complejidad es que "la metacognición como herramienta interna del sujeto que aprende dialoga internamente con su "yo-interno"; toma conciencia de la visión metacompleja (pensamiento complejo-metacognición) de lo que investiga" (GONZÁLEZ, 2017, p.31).

Estos procesos son verdaderos ejercicios del pensar profundo con la matemática; sigamos los topois, separabilidades y procesos mentales creados por el dominio colonial en la enseñanza de la matemática que en la transdisciplinariedad de disuelven de la concepción reduccionistas: concreto-abstracto, análisis-síntesis, inducción-deducción, 


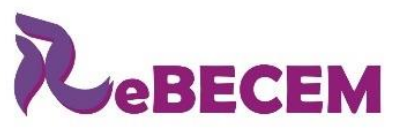

Revista Brasileira de Educação em
Ciências e Educação Matemática

DOI: https://doi.org/10.33238/ReBECEM.2020.v.4.n.4.24986

verdad-error, absoluto-relativo, teoría-práctica, macro-micro (PEÑALVA ROSALES, 2010). Vamos brevemente en un ejercicio que da apertura, como exprese puede ser motivo de estudios de tesis doctorales.

En lo concreto-abstracto la matemática, "lo concreto y lo abstracto no pueden separarse; son dos aspectos solidarios, dos caracteres inseparables del conocimiento que, sin cesar, pasan del uno al otro" (PEÑALVA ROSALES, 2010, p.138). Lo concreto se ha execrado del proceso de la matemática, se pretende que sea abstracto cayendo en la imposibilidad de ir en espaciado mental desde lo cotidiano a la abstracción, en los primeros niveles de educación. Aceptar la inseparabilidad de lo abstracto con lo concreto es ir a estadios del pensamiento profundos, con el proceso de inseparabilidad: concretoabstracto-concreto.

En la matemática, el pensamiento concreto en los procesos lógicos matemáticos va al: análisis, síntesis, generalización, así como extraer conclusiones sobre de los objetos y fenómenos; pero al mismo tiempo se transita a lo concreto sobre la base de la práctica y comprende, esencialmente, procedimientos como el análisis y la síntesis. "Los conceptos más abstractos y más iniciales sirven para explicar los más tardíos y más concretos" (CALLINICOS, 2014, p. 130).

En el proceso análisis-síntesis en la matemática:

La síntesis se manifiesta como complementaria al análisis. La síntesis se define, en general, como una operación (...) por medio de la cual se rehace en sentido inverso el camino recorrido por el análisis. La síntesis reconstruye el todo, asegurándose de no omitir nada (PEÑALVA ROSALES, 2010, p.139).

Cuando estudiamos matemática sabemos que nos vamos a separar para comprender y luego unificar para ver el todo de la construcción; no existe análisis sin síntesis, y no se hace síntesis sin el análisis.

Con el análisis-síntesis estudiamos problemas complejos, y no podemos quedarnos estacionados en la síntesis vamos al análisis y volvemos a unir las partes y regresamos al todo, la perspectiva compleja en todo momento. El análisis-síntesis forma parte del hacer matemático ocultado muchas veces en el análisis por un lado y la síntesis por otro lado; al fin ninguna sin la otra se desarrolla de manera completa en el pensar.

La diada inseparable en la matemática: inducción-deducción, "inducción y deducción nos llevan respectivamente de la realidad a la teoría, y de ésta a la práctica" (PEÑALVA ROSALES, 2010, p.140); no hay teorías que en matemática no vayan a la 
DOI: https://doi.org/10.33238/ReBECEM.2020.v.4.n.4.24986

ejemplificación a la ejercitación; el ejemplo, los contraejemplos son cotidianidades del hacer matemática que en la lógica dialéctica son importantes y no se separan.

Los procesos inducción-deducción son procesos mentales de alto nivel usado en la filosófica aristotélica, desde luego antes que Grecia perdiera su preminencia de complejidad en la creación del conocimiento en tanto, luego al ser invadida por Roma (DLUGOSZ, 1873). Aquí quiero hacer un previo, para comprender el cómo se presenta la soslayación en los procesos de pensamiento del ser humano (PLATON, 2012).

Nosotros, no hemos sido, los del Sur, los únicos invadidos y soslayados; en el construir del conocimiento la invasión marco patrones de hacer ciencia, de enseñar, entre otras. El conocimiento antiguo, el conocer por el mero disfrute de desarrollar el intelecto, de tener conversaciones de altura entre filósofos, matemáticos; el abrazo de la filosofía con Paltón y la matemática con Pitágoras, también en la historia fue disuelta en las mentes de los futuros profesionales, en cuanto Roma, la estricta, la de la milicia y el don mando invade a la romántica Grecia, siglo III a.C, difícil es dilucidar el alcance que Roma, pudo desplegar en el arte griego, en la ciencia a partir esa época, hasta el final en teoría del periodo helenístico, el año 30 a. C.; la:

Ruina de Constantinopla, tan funesta (...) el nombre de Cristo insultado y envilecido. (...) Con las bibliotecas quemadas y los libros destruidos, la doctrina y la ciencia de los griegos, sin las que nadie se podría considerar sabio, se desvaneció (DLUGOSZ, 1873, p.532).

Es así como un hecho tan marcado da un salto de opresión donde actualmente la lógica dialéctica no es usada en la matemática para los procesos de deducción-inducción:

La inducción que lleva a cabo el intelecto (...) sirve para la adquisición de los principios prácticos universales a partir de los principios prácticos particulares. Ambos son útiles para el agente moral, pero la diferencia entre ellos está en que los universales son necesarios para la virtud completa y suprema (MARES, 2015 , p.48).

Que el talento en las instituciones educativas se pierda de los procesos mentales profundos de la matemática de deducción-inducción, es lamentable; los procesos de pensamiento por excelencia de los teoremas, lemas, axiomas; y que en la enseñanza de la matemática no se lleven a cabo como significa es porque en esencia la separación de la filosofía marca procesos soslayadores que le quitan el proceso correcto de la deducción y la inducción para llegar al pensamiento profundo.

El gran matemático George Papy, se pregunta: 
DOI: https://doi.org/10.33238/ReBECEM.2020.v.4.n.4.24986

¿Por qué se pide a la matemática que forme individuos que sólo tomen en cuenta la eficacia instrumental de las matemáticas? La matemática es un arte ligado a lo más profundo del hombre, y es también educación (PÉREZ, 1980, p.43).

La matemática por excelencia, en el desarrollo de la mente, el pensar profundo destinado escuetamente, denegridamente a técnicas mecánicas e instrumentos. El arte religado a estructuras profundas del ser humano en el pensar es condición urgente a regresarle a la matemática en la vida de las personas; y provocar descubrir la razón en el individuo.

La diada inseparable en la matemática: verdad-error, la imposición de verdad arbitraria en la matemática le quita la belleza al error, "todo error puede ser en sí mismo una verdad parcial o el aspecto de una verdad. Todo error permite ampliar los límites de una verdad, negándola inicialmente" (PEÑALVA ROSALES, 2010, p.140); por ello en matemática existen lo que denominamos condiciones iniciales, en donde una verdad es así en tanto no se rompan esas condiciones iniciales; así como lo que es allí no es totalmente cierto en la naturaleza, por ejemplo; en esta no conseguimos figuras geométricas perfectas, pero la regularización que hacemos en el aula ayuda a la comprensión en la naturaleza.

El proceso de argumentar está presente en todos los momentos la matemática, es un proceso de alto nivel cuando de afirmar algo se trata, el ¿por qué? se quiere garantizar la verdad o falsedad de ciertas afirmaciones, bajo ciertas condiciones. Este proceso puede llevar a preparase para argumentar en la vida ante una situación, de allí que la matemática en la con-formación del ciudadano tiene pleno sentido (RODRÍGUEZ, 2013). En este aspecto para efecto de verdad o error:

Verificar la propiedad general en muchos ejemplos podría ser un argumento válido, mientras que en un contexto de formación de profesores en el área de Aritmética, un argumento válido sería una demostración por inducción (ÁLVAREZ et al., 2013, p.82).

La diada inseparable en la matemática: absoluto-relativo:

Las verdades "absolutas" - aceptadas, aunque temporalmente, por la humanidad para construir sobre ellas las explicaciones del mundo - se alcanzan por medio de los descubrimientos relativos y de los pensamientos individuales, cuyo alcance es limitado (PEÑALVA ROSALES, 2010, p.140).

La superación de lo relativo para ir a lo absoluto y conseguirle relatividad a lo absolutista son procesos complejos de la matemática que bien dirigido desde los procesos 


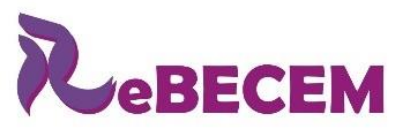

Revista Brasileira de Educação em

Ciências e Educação Matemática

DOI: https://doi.org/10.33238/ReBECEM.2020.v.4.n.4.24986

más elementales a lo de más alto pensar profundo pueden desarrollar estadios del pensamiento deseable para aprender a pensar, y re-ligar el pensar.

La diada inseparable en la matemática: teoría-práctica, la matemática convive entre teorías y practicas; entre lo que marca la ejemplifican; al ser parte de este mundo que se quiere comprender, "para conocer los objetos será necesario actuar sobre ellos. Sólo así se puede garantizar de alguna manera que el conocimiento adquirido es relativo respecto del lugar que ocupamos en el universo" (PEÑALVA ROSALES, 2010, p.140).

Es así como, "la noción de que la promoción del aprendizaje por parte del maestro se puede operar en términos de la transgresión de normas en situaciones y contratos, permite asimilar la promoción de los aprendizajes a una teoría de la práctica" (HERBST, 2018, p.33). Trasgrediendo las normas en el concepto matemático, en el discernir se pueden entender la necesidad de la norma, de esa condición inicial del problema; ¿por qué no podrá dividir por cero? ¿Cómo se delinea esa imposibilidad en la mente?; ¿se acepta como una prohibición o se discierne sobre su imposibilidad?

La diada inseparable en la matemática: macro-micro, hay que tener clarificado que "es necesario observarlo de manera alternativa desde el punto de vista social (de sus rasgos generales) y desde el punto de vista privado. Esta doble observación permite alcanzar lo universal que puede ser aplicado al hombre y la riqueza de lo particular" (PEÑALVA ROSALES, 2010, p.141). Es notorio, pero no siempre evidenciado, que la dicotomía entre lo macro y lo micro no es pertinente, no es verdad, uno no existe sin el otro. La diferencia micro macro no está establecida en la matemática, no hay general sin lo particular y no hay particular sin lo general.

Pero la tradicionalidad separo la díada: micro-macro; y ello le ha hecho mucho daño a la matemática en la enseñanza así como la constitución de otras disciplinas como la economía; "la diferenciación entre micro y macro no es pertinente. Y si es válida, queda sin piso la pretensión de micro fundamentar la macro" (GONZÁLEZ, 2004, p.74); sólo se ha diferenciado en el pensar disyuntivo más no en el profundo.

Se quiere terminar afirmando y haciendo un barrido al hecho de aceptar la imposibilidad de la matemática impuesta en la vida del niño; causando graves problemas que más adelante se presentan en dificultades de aprendiza; y que reversamente una matemática bien llevada en el niño puede ayudar a superar problemas de aprendizaje y de personalidad; no es una panacea lo que aquí afirmo, le incito a los negadores de estas realidades a navegar en el arte de pensar profundo diluyendo su falsas concepciones en la 
DOI: https://doi.org/10.33238/ReBECEM.2020.v.4.n.4.24986

investigación de la temática, no pierdan esa oportunidad, nuevamente vuelvo con el matemático y pedagogo George Papy:

Los niños o individuos que han estado bloqueados para aprender matemáticas, han estado bloqueados también en su personalidad. Un niño que no aprendió matemáticas se siente disminuido en sí mismo como individuo. Se puede hablar, pues, de una relación profunda entre el conocimiento matemático y la personalidad (PÉREZ, 1980, p.44).

Vamos a atacar esa realidad con un re-ligar del pensamiento, en Rodríguez (2019b), se afirma de cómo enseñar matemática des-ligando nuestra principal problema como docente que impide desarrollar plenamente el pensamiento en nuestros discentes en la enseñanza; y aunque se sientan irónicamente aquellos docentes liberados haciendo un ejercicio antiético bajo el autoritarismo ellos también están oprimidos; no hay posibilidad de ser opresor sin estar oprimido. Todo ello ha sido estudiado debidamente en la pedagogía del oprimido de Paulo Freire.

\section{Rizoma: Estrategias metacognitivas complejas, como emergentes re-ligantes en la enseñanza de la matemática}

Se trata en este rizoma se analizan estrategias metacognitivas compleja, como emergentes re-ligantes en la enseñanza de la matemática. Se asumen las estrategias metacognitivas un proceso de madurez intelectual-espiritual-emocional del ser humano, ya que conocer y reflexionar sobre los propios procesos mentales y buscar estrategias remediales ante las dificultades comprensivas, exige una autonomía de pensamiento propia del pensamiento abstracto (POGGIOLI, 1998).

Que, ¿si se puede enseñar a pensar?, más que eso, enseñar la condición humana desde el inmenso potencial que tenemos a la imagen creadora de Dios, un ser complejo capaz de inmensas realizaciones con el pensar profundo ante una responsabilidad de que hacemos con el otro, como si fuéramos nosotros mismos, en actos de solidaridad y bondad “enseñar a autorregular la actividad mental es lo mismo que enseñar estrategias efectivas de aprendizaje, el desarrollo de la metacognición da como resultado saber aprender" (PEÑALVA ROSALES, 2010, p.145). No es de extrañar el hablar de Dios y las creencias de la autora, en vista que ya se dijo que es partícipe de la investigación, sujeto en liberación por el transmétodo la deconstrucción rizomática. Tengan en cuenta respetados 


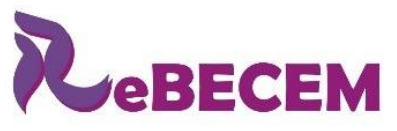

\section{Revista Brasileira de Educação em \\ Ciências e Educação Matemática}

DOI: https://doi.org/10.33238/ReBECEM.2020.v.4.n.4.24986

lectores que esta es una investigación enmarcada en el proyecto liberador transmoderno; donde el sujeto es agente de cambio del proceso. La intencionalidad es liberadora.

Se trata de desarrollar el pensamiento complejo niveles de procesos mentales de alta metacognición; entonces las estrategias complejas son estrategias para el desarrollo del pensamiento complejo; que se hace consiente en el hacer y el pensar profundo. Y eso no se puede lograr si no se provoca la tetra: cuerpo-mente-alma-espíritu. Por ello, hablar de estrategia mentales, bajo la concepción que sólo la mente colabora en tal hecho es falso.

Se quiere dilucidar que listar una cantidad de actividades instrumentales de hacer para supuestamente aprender matemática, sin hacer consciente el valor de aprender a pensar; pensado que memorizar mecánicamente es el fin, sería caer en el pensamiento parcelado en acomodadizo a niveles separado y ya sabemos que así no ha dado resultado el pensar profundo.

Es urgente en todo momento conectar la enseñanza y el aprendizaje con emociones positivas, algo fundamental para alejar a los discentes del bloqueo y el hastío para ello, el docente debe ser portador del yo sí puedo en el yo sí puedo del educando; de la alegría por el mero disfrute de aprender fuera de las amarras de la evaluación y de la aprobación de un contenido. En ello es esencia el respeto y la valoración humana de ese discente que cree en el docente y dedica su tiempo a que en otro en igual condición de respeto y amor marque su vida positivamente (RODRÍGUEZ, 2013).

La innovación, creatividad, fomento del hacer inmiscuyendo la motivación en ambientes de aprendizaje; acá debe hacerse evidente el conocer como el discente aprende; la generalidad de que todos aprende de la misma manera es errónea, en ese explorar jugando al geómetra con la alegría, en un ir y venir paseándose por el lenguaje el significado de sus símbolos, las diversas interpretaciones; soltar las amarras de conseguir la solución del problema como la meta es bueno, es el disfrute del procedimiento, de las diversas posturas que se van presentando.

Esos encuentros deseados donde se quiere volver al grupo, a disfrutar de cómo se hacer debe ser provocado por el docente; aceptar la diversidad de pensar de su grupo; ir evaluando como cada quien va elevando ese pensar y cada vez va creyendo al mismo tiempo que si puede lograrlo; entonces el docente es un entusiasta por la ciencia, un convencedor de oficio, un artista del color; un transdisciplinador que conoce las conexiones ocultas que relacionan el conocimiento con las diversas ciencias de ese objeto 


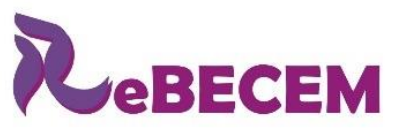

Revista Brasileira de Educação em

Ciências e Educação Matemática

DOI: https://doi.org/10.33238/ReBECEM.2020.v.4.n.4.24986

matemático que lo devela en el aula como objeto transdisciplinar y lo lleva al escenario nutriéndolo cada vez más de ideas innovadores de aportes a la humanidad.

Ese emocionar va calando en el discente, y cada uno se vuelve multiplicador con su compañero; donde el aventajado en tiempo para hacer, gana al que todavía no consigue el ancla que lo lleve al conocer; es bueno allí romper la distancia docente-estudiante; sino que en grupos colaborativos lo van logrando, ascendiendo al objeto transdisciplinar; así es denominado el objeto matemático transdisciplinado por la colaboración de los conocimientos de cualquier disciplina. En todo ello debe haber un dialogo con el yo interno de los actores del proceso educativo y debe quedar develado como en ese juego de palabras pasan en movimientos: abstracto-concreto, micro-macro, teoría-práctica; entre otros.

Aprender de lo concreto a lo abstracto permite desarrollar estrategias; pero debe soltarse por mucho tiempo las amarras de la perfección en la construcción; sin embargo ir ascendiendo a ella; en todo momento debe haber retroalimentación acudiendo a la historia y filosofía de la matemática a la lógica dialéctica de cómo pensar por el mero hecho de entrenar el cerebro; provocar en el con las diferentes anécdotas y circunstancias por donde los inventores del cálculo, por ejemplo, fueron pasando para develar en símbolos tan magnifica perfección de Dios en la naturaleza.

En las diversas pruebas del teorema de Pitágoras existe lo místico, la intuición; en tanto como lo místico de devela en el aula con pruebas que ascienden 367 diversas maneras de probar el teorema documentas en el libro de Elisha Scott Loomis de 1927 denominado: The Pythagorean Proposition. Ya actualmente se pasan de mil diversas maneras de probar el teorema; algunas pruebas geométricas tan hermosas, sin usar un solo número. Hay que enamorar el estudiante con ese juego que con el Tangram, un rompecabezas muy antiguo y de origen chino, que se puede usar para lograr el movimiento de las piezas de cualquier material, donde se van formando figuras (IGLESIAS, 2009).

Así mismo, en la enseñanza los metamétodos heurísticos complejos deben pasar primeramente por los procesos del lenguaje de la matemática, y del lenguaje en sí de la comprensión de lo que se quiere hacer en el proceso: señalar las ideas más transcendentales, contrastar ideas, identificar los puntos de controversia, identificar relaciones, investigar palabras clave, señalar diferentes aspectos del tema o concepto que se han considerado, revisar datos, esquemas, figuras, es una variedad posibilidades antes de intentar ir a ese número o esos números de soluciones al problema que el docente se 


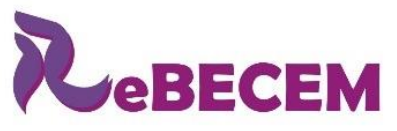

Revista Brasileira de Educação em

Ciências e Educação Matemática

DOI: https://doi.org/10.33238/ReBECEM.2020.v.4.n.4.24986

propone obtener que lo llevan desquiciadamente a desvalorizar el procedimiento en sí, su comprensión su justificación desde el estudiante; así los ¿Por qué? deben hacer parte de las preguntas diarias del proceso que lleve al pensar profundo.

Se deben promover en la enseñanza estrategias complejas y transdisciplinares para el desarrollo de la investigación transmétodica; más allá de los pasos memorísticos intocables de la tradicionalidad, para ello, la investigación transdisciplinar crítica, como transmétodo, debe enseñarse más allá de la matemática, pero con la matemática; pensando dialogando; ese dialogo profundo al estilo Sócrates, Platón elevando el arte de pensar a estadios hermosos de la duda, del rebatir una idea errónea con pocas palabras; el contraejemplo deviene siempre de un pensar profundo.

La díada: ecosofía - diatopía debe ser centro de la enseñanza; dale preminencia al formarse para aprender el difícil arte de habitar en el planeta; por ello la con-formación de ese ciudadano debe aprovecharse para ir delineándole libremente en el aula; evitando el estudiante sumiso al que hay que despertarle su valor y sentido de ser en la humanidad; un ciudadano del mundo que viene a marcar su huella. Eso puede hacerse, con un docente formado en tal excelsitud. De la diatopía, podemos comenzar a promover la complejidad con esos diálogos de saberes que van comunicando lo disímil en apariencia en el aula. La matemática es prueba y valor de la comunicación y la concreción de la teoría de la complejidad.

El valor de los conjuntos y la lógica dialéctica debe colaborar en todo momento regresándose y recreándose en ella a aprender: dos conjuntos pueden ser disjuntos, pero aun así pese a que no se comunican con elementos comunes lo hacen con el elemento vacío que sabemos que pertenece a cualquier conjunto; ello tiene una significancia unitiva en la vida que rompe con los topois y deviene en el pensar profundo del estudiante el hermoso arte del pensar con al excelsitud de la matemática.

El número uno (1) como elemento factor común entre varias expresiones algebraicas es sentido indicativo de la unión universal, de lo indivisible e indecible de la humanidad. Si no hay factor común siempre existirá ese uno número factor común, que les recuerda la unicidad de la vida; la responsabilidad ante el otro en ese accionar pues la comunicación invisible muchas veces, intangibles es realidad eterna de creación del universo; la huella de amor de Dios, en el debemos atender con proceso dialógicos profundos en la enseñanza.

El enseñar debe ser un arte de hacer investigación transdisciplinar crítica, colaborando a producir conocimientos transdisciplinados sólidos, a partir de considerar 


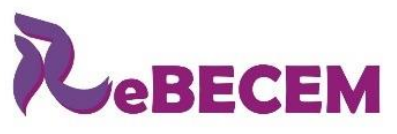

Revista Brasileira de Educação em

Ciências e Educação Matemática

DOI: https://doi.org/10.33238/ReBECEM.2020.v.4.n.4.24986

los contextos y las vivencias que se producen en el mundo real; provocando una ecología de los saberes en los discentes; por ello, en una aula mente social permanente, el espacio intersubjetivo de aprendizaje, el arte de aprender matemática con la vida debe ser promovido por el docente; explorando la experiencia de sus estudiantes, buscando en su cultura, en su cotidianidad y desde lo concreto formalizar lo abstracto en el aprendizaje.

En los primeros niveles educativos, las universidades deben haber formado docentes para que en esos niveles ellos sean capaces de entender conceptos complicados con la experimentación del estudiante y que puedan visualizar en todo momento lo que están haciendo y disfruten del proceso. No se dice que el proceso entero de la conceptualización de la matemática se formalice con la cotidianidad; digo que allí en la naturaleza, en el juego del niño hay delineados logaritmos, exponenciales; entre otras; que despiertan el interés. Que no decir de la bella geometría tan esencial esos niveles para provocar el pensar profundo; cuando los estudiantes lleguen a nivel universitario ese pensar profundo se eleva sin dificultad hacia estadios mayores de abstracción.

Las complejidades educativas de la matemática con el uso de las tecnologías son clarificadas con menor dificultad, ¿Por qué un estudiante prefiere muchas veces los computadores para estudiar? Estimados docentes porque las computadoras no castigan el error, dan un aviso y casi siempre en ese proceso el estudiante se regresa a aprender; además que las tecnologías le dan una variedad compleja de posibilidades de aprender recreándose. He ahí un punto de aversión al docente que debe ser clarificado en la enseñanza, mostrándose humano, amigo, ser humano que abraza, que se preocupa por el sentir de ese ser que está allí inerte castigado disminuido en un proceso que se le hace interminable. Por ello, el docente se debe volver un experto computista, ¿Cómo hacerlo? Por favor, aprovechen sus aventajados estudiantes en las tecnologías y convóquenlos desde ese espacio a las diversas formas de aprender matemáticas; son muchísimas: videos de la historia de la matemática, juegos; entre tantos más. Con ello aprenderán a que fuentes del Internet no consultar, entre otras; a discernir en vez de repetir; a clasificar en vez de copiar y pegar.

Es urgente la formación del docente de matemáticas, comprender que el conocimiento matemático es permanece abierto, contingente e incierto, que convoca a asumir una actitud de inacabado en el mar de la complejidad del conocer. De ello, deviene lo abrazador e inclusivo; donde lo único que excluye es lo que delimita lo excluyente; convocar al abrazo de los saberes en igual grado de importancia, legitimando los de la periferia; hablo de lo encubierto, lo soterrado lo desvirtuado donde la esencia de lo 


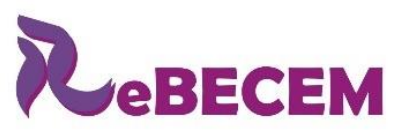

Revista Brasileira de Educação em

Ciências e Educação Matemática

DOI: https://doi.org/10.33238/ReBECEM.2020.v.4.n.4.24986

concreto puede llevarse a lo abstracto en un pensamiento profundo. Develar en la formación en que momento la matemática es su arma de soslayación, como fueron formador, bajo ese ejercicio antropoético los docentes están en la oportunidad de re-ligar des-ligando su propia formación; el ejercicio de conciencia también en un pensar profundo les haría mucho bien al ser opresivo dentro de su formación reduccionista, parcelada.

La investigación transdisciplinar crítica en la enseñanza de la matemática debe ser llevada a la enseñanza; pues es descolonizadora planetaria de los saberes matemáticos; llevando los pertinentes al aula, que las realidades que allí se estudiante sean realidades de interés cotidiana y cultural del discente. Bajo esta característica no hay centrismo en la producción del conocimiento matemático transdisciplinar; sino multicentrismo, es decir, como estrategia, tomando en cuenta que en conocimiento, los saberes en sí mismo no tienen sesgos; sino que los representantes de la disciplinas los sesgan y producen los centros.

La matemática no es sólo cantidad, sino cualidad, critica, pensar profundo en un hacer del pensamiento que no debe disolverse o remitirse a un algoritmo; ellos son sólo herramientas no el centro y el hacer de la matemática; la investigación transdisciplinar crítica que se propende como manera de hacer matemática, y que nuestros estudiantes pueden hacer es complementaria, donde los enfoques sean cualitativos-cuantitativossocio-críticos, y plurales, pues en las fronteras de los conocimientos consiguen esencias complejizadoras.

Aprovechando la transdisciplinariedad profunda de la matemática con todas las ciencias y los saberes esta realidad debe ser llevada a la enseñanza; sentires en conferencias de la autora en el III Congreso Nacional sobre Didáctica de la Física y el I Congreso Nacional sobre la Didáctica Transdisciplinar de las Ciencias, Araya Estado Sucre, Venezuela, 2014, titulada como: la didáctica de la física y la didáctica de la matemática: un noviazgo profundamente transdisciplinar; muestran el concierto de fantasías entre la física y la matemática; grandes relaciones casi desconocidas en el estudiante: Menecmo y Apolonio de Perga y la historia de las cónicas, la parábola en todas partes, Pitágoras de Salmos en la historia, el teorema llamado de Pitágoras: 4000 años de historia geométrica, ejemplos con el Teorema de Pitágoras y problemas de física, Arquímedes de Siracusa es el vivo retrato del físico-matemático, Isaac Newton intento casar a los novios (la física y la matemática), “'existió la manzana de Newton?, Albert Einstein, el más grande físico humanista; entre tantas relaciones transdisciplinares por 


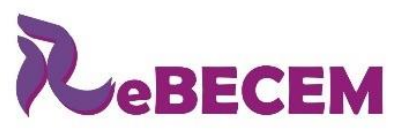

Revista Brasileira de Educação em

Ciências e Educação Matemática

DOI: https://doi.org/10.33238/ReBECEM.2020.v.4.n.4.24986

mostrar; tarea pendiente que indagando e innovando es posible" (Rodríguez, 2020d, p.12). Son tantas las posibilidades para enseñar, que no se justifica se siga de la misma manera

Si un cuerpo es irregular, una piedra por ejemplo, no existe una fórmula matemática que permita determinar su volumen, y si la hay de seguro que es muy compleja, ¿Qué ideas innovadoras se tienen para calcular el volumen de esa piedra? Parece capcioso preguntar ¿por qué un barco flota a pesar que es de metal y el metal tiene mayor densidad que el agua? Un submarino se hunde o flota a discreción: ¿cómo lo hace? Todo ello debe interesar al docente de matemática, ¿Qué tienen de especial los peces que hace que floten y se hundan? Los peces se sumergen o se elevan en el agua inflando o desinflando su vejiga natatoria. Con experimentos sencillos a los niños se les puede motivar (Rodríguez, 2020c). La validez de las teorías no puede discutirse sólo en base a los conocimientos actuales, sino sobre todo a partir del contexto en el que surgen, sus complejidades y esencia. Es imbuirse en el corazón de sus creadores, su sentir.

El desarrollo del pensamiento profundo físico-matemático, el noviazgo que le he descrito, corresponde a uno de los objetos de estudio de la didáctica física y de la matemática, pero también del matemático y del físico que ejerce la docencia a partir del cual el estudiante construye y da significados a los diferentes fenómenos físicos por medio de la matematización:

Se debe ir al desarrollo del pensamiento profundo físico - matemático complejizado: numérico-lógico- critico- espacial- métrico- aleatoriovariacional. El uso de los conceptos y teorías de la física y de la matemática deben ser considerados como una herramienta de trabajo dentro y fuera del aula desde donde formar habilidades de pensamiento en los estudiantes y no como la meta a conseguir (RODRÍGUEZ, 2020d, p.13).

El recorrido por las estrategias complejas en el re-ligar de la enseñanza de la matemática no es estricto, ni lista de tareas; es una meseta que se anida desde la investigación que continua, con cierres que son aperturas en el rizoma siguiente.

\section{Rizoma de aperturas al pensamiento profundo}

En esta investigación transmetódica con la deconstrucción es espacios transmodernos de ha analizado la tríada: metacognición-complejidad-matemática para 


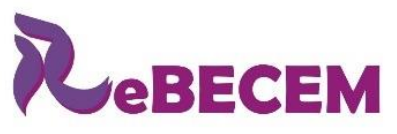

Revista Brasileira de Educação em

Ciências e Educação Matemática

DOI: https://doi.org/10.33238/ReBECEM.2020.v.4.n.4.24986

dar esencias que vayan al pensar profundo en el aula valorando la matemática en toda su complejidad.

En la línea de investigación: Educación Matemática Decolonial Transcompleja, se continúa con la investigación en las estrategias complejas re-ligantes; hemos caracterizados los procesos de aprendizaje en el cerebro y sólo allí, la metacognición; esa es la tradicionalidad; pero si consideramos la tetra: cuerpo-mente-alma-espíritu como la esencia del ser humano es lógico pensar que aportes sustanciales de lo que hace y aprende debe esta supeditados, desde la esencia de la consideración compleja del ser humano; debe estar supeditado a esos cuatro aspecto de la tetra que no se desunen; todos estos estudios se continúan en la mencionada línea de investigación titulada: Educación Matemática Decolonial Transcompleja.

La autora quiere volver sobre el titulo de la investigación: la matemática en la metacognición o la metacognición en la matemática: metacognición - complejidad matemática. Quiera decir que la tríada: metacognición-complejidad-matemática desde el titulo; para quienes discernimos rápidamente ya estamos diciendo que las categorías: metacognición, complejidad, matemática no se desunen. Y que claramente la matemática interviene en la metacognición y la metacognición en la matemática; siempre y cuando la matemática sea enseñada en su complejidad, sin soslayaciones; la decolonialidad sea eje constitutivo de su educación. No hay posibilidad de afección unidireccionalidad en la complejidad; es una bidireccionalidad; si una es afectada por la otra; esta otra afecta a la primera.

Ahora, pensando en las inteligencias múltiples como una complejidad, en especial algunas que están en una escala, si se puede hablar de ello, más elevadas que la lógico matemática, refiero a la emocional y espiritual; ¿qué parte del aprendizaje de las matemáticas, los procesos matemáticos, depende de estas inteligencias? ¿O seguimos pensando que la inteligencia lógico-matemática es la única que aporta al ser humano sustancia para aprender matemáticas? ¿Dónde se ubica la conciencia?

Ahora para investigar tales interrogantes, desde el pensar profundo que es complejo y transdisciplinar el docente de matemática debe preguntarse en primer lugar: ¿qué es la inteligencia espiritual? Ya hay antecedentes de la indagación y grandes experiencias vividas por matemáticos antiguos hasta hoy, LUQUE (2011) en su tesis doctoral titulada: Una metodología para la enseñanza de las matemáticas en secundaria basada en las inteligencias emocional y espiritual, afirma que las inteligencias emocional y espiritual, mejoraría sensiblemente el rendimiento académico de los discentes. ¿Qué 
DOI: https://doi.org/10.33238/ReBECEM.2020.v.4.n.4.24986

estrategias complejas re-ligantes del proceso de enseñanza se pueden considerar? ¿Cuáles son los cambios des-ligados de la tradicionalidad se deben llevar con la aceptación de tal realidad a la enseñanza? Es de advertir; que el investigador debe estar re-ligado a pensamientos profundo del ejercicio ético de ser, parecer y hacer; esto es si no puede desarrollar una inteligencia espiritual elevada se distanciará emocionalmente de la indagación y de dedicará a dar instrumentos y estrategias disyuntivas que hasta ahora no han calado.

El camino del conocer, de pensar profundo, un proceso metacognitivo de alto nivel también es subjetivo por ser complejo e intervienen todas las dimensiones del ser humano; es una meseta hermosa que vale la pena siempre transitar. Como matemático, como mi ejercicio favorito de pensar profundo siempre afirmo: cuando estudio matemáticas mis pensamientos se eleva hasta el universo, y sus similitudes de perfección con esté me hacen entender el poema más bello que de la misma vida se hace. Al final, comienzo y fin del pensamiento profundo; el máximo nivel de la inteligencia espiritual es la sabiduría que sólo tu Espíritu Santo de mi Dios amado, Matemático por excelencia magnifica, nos da: "la exposición de tus palabras alumbra; hace entender a los simples" (Salmos 119: 130). Amén.

\section{Referencias}

ÁLVAREZ, I.; BAUTISTA, L.; CARRANZA, E.; SOLER, M. Actividades Matemáticas: Conjeturar y Argumentar. Números, Madrid, v.85, p.75-90, 2013.

CALLINICOS, A. Deciphering Capital. Marx's Capital and its Destiny. Londres: Bookmarks Publications, 2014.

DELUEZE, G.; GUATTARI, F. Mil mesetas. Capitalismo y esquizofrenia. Valencia: Pretextos, 2004

DLUGOSZ, J. Historiae polonicae libri XII. Vol. 1. Krakow: Edited by Alexander Przeździecki, 1873.

DUSSEL, E. La producción teórica de Marx: un comentario a los Grundrisse. México: D.F., Siglo. XXI, 1985. 
DOI: https://doi.org/10.33238/ReBECEM.2020.v.4.n.4.24986 ISSN 2594-9179

DUSSEL, E. Transmodernidad e Interculturalidad: Interpretación desde la Filosofía de la Liberación. En E. Lander (comp.) La colonialidad del saber: Eurocentrismo y ciencias sociales. Perspectivas latinoamericanas. Buenos Aires: Unesco, Ciccus, Clacso, 2005.

FERNÁNDEZ, J. Neurociencias y Enseñanza de la Matemática. Prólogo de algunos retos educativos. Revista Iberoamericana de Educación, Madrid, v.51, n.3, p.1-12, 2010.

GONZÁLEZ, J. La dicotomía micro-macro no es pertinente. Revista de Economía Institucional, Universidad Externado de Colombia, v.6, p.73-95, 2004.

GONZÁLEZ, J. Teoría educativa transcompleja. Barranquilla: Universidad Autónoma del Caribe, 2017.

HERBST, P. Teoría y métodos para la investigación de la racionalidad de la práctica en la enseñanza de las matemáticas. Educación matemática, México, v. 30, n.1, p.9-46, 2018.

IGLESIAS, M. Ideas para Enseñar. El Tangram en la Enseñanza y el Aprendizaje de la Geometría. UNION Revista iberoamericana de Educación Matemática, Madrid, v.17, p.117126, 2009.

LEIBNITZ, G. La monadología. Opúsculos. Traducción de Antonio Zozaya. Madrid: Plaza del Progreso, 1889.

LUQUE, V. Una metodología para la enseñanza de las matemáticas en secundaria basada en las inteligencias emocional y espiritual. (Tesis Doctoral). Universidad Nacional de Educación a Distancia: España, 2011.

MARES, E. La inducción como método de conocimiento de los principios éticos en la Ética nicomáquea de Aristóteles. Diánoia, México, v.60, n.75, p.31-53, 2015.

MONTAIGNE, M. Los ensayos (según la edición de 1595 de Marie de Gournay). Quinta edición. Barcelona: El Acantilado, 2007.

MORÍN, E. La Cabeza Bien Puesta: Repensar la reforma. Reformar el pensamiento. Buenos Aires: Nueva Visión, 1999.

MORÍN, E. Introducción al pensamiento complejo. Barcelona: Gedisa, 2005a.

MORÍN, E. Epistemología de la complejidad. En: Biblioteca Virtual sobre el pensamiento complejo. Recuperado de: www.pensamientocomplejo.com, consultado el 9 de mayo de 2020, $2005 b$. 


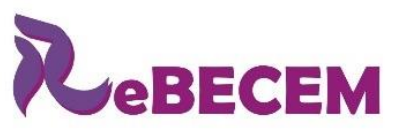

Revista Brasileira de Educação em

Ciências e Educação Matemática

DOI: https://doi.org/10.33238/ReBECEM.2020.v.4.n.4.24986

OLANO, A. Estudios internacionales latinoamericanos y pensamiento decolonial.

Contribuciones a un conocimiento situado. Bogotá: Universidad Externado de Colombia, 2019.

ORTIZ, A.; ARIAS, M.; PEDROZO, Z. Pedagogía decolonial: hacia la configuración de biopraxis pedagógicas decolonizantes. Revista Ensayos Pedagógicos, v. XIII, v. 2, p.1-15, 2018.

PEÑALVA ROSALES, L. Las matemáticas en el desarrollo de la metacognición. Polít. cult., México, n. 33, p. 135-151, Jan. 2010. Disponible en

<http://www.scielo.org.mx/scielo.php?script=sci_arttext\&pid=S0188-

$77422010000100008 \& \operatorname{lng}=$ es \&nrm=iso > . accedido en 11 nov. 2020.

PÉREZ, A. Las matemáticas modernas: pedagogía, antropología y política. Entrevista a George Papy. Perfiles Educativos, México, v.10, p.41-46, 1980.

PLATÓN. Diálogos. México D.F.: Porrúa, 2012.

POGGIOLI, L. Estrategias metacognoscitivas. Caracas: Fundación Polar, 1998.

RODRÍGUEZ, M. La matemática: ciencia clave en el desarrollo integral de los estudiantes de Educación Inicial. Revista Zona Próxima, Barranquilla, v.13, p.130-141, 2010.

RODRÍGUEZ, M. La educación matemática en la con-formación del ciudadano. TELOS.

Revista de Estudios Interdisciplinarios en Ciencias Sociales, Maracaibo, v.15, n.2, p.215 230, 2013.

RODRÍGUEZ, M. Re-ligar como práctica emergente del pensamiento filosófico transmoderno. ORINOCO Pensamiento y Praxis, Ciudad Bolívar, 11, p.13-3, 2019a.

RODRÍGUEZ, M. Deconstrucción: un transmétodo rizomático transcomplejo en la transmodernidad. Sinergias educativas, Ecuador, v.4, n.2, p.1-13, 2019b. DOI:

https://doi.org/10.31876/s.e.v4i1.35

RODRÍGUEZ, M. La educación matemática decolonial transcompleja como antropolítica.

Utopía y Praxis Latinoamericana, Maracaibo EXTRA 4, p. 125-137, 2020a. DOI:

http://doi.org/10.5281/zenodo.3931056

RODRÍGUEZ, M. La alfabetización matemática en profesionales universitarios: imposibilidad o re-significación urgente. Revista Innovación Educativa, v. 2, n.2, p.226-244, 2020b. DOI: https://doi.org/10.35622/j.rie.2020.02.001 
DOI: https://doi.org/10.33238/ReBECEM.2020.v.4.n.4.24986

RODRÍGUEZ, M. Serendipiando con los procesos mentales de la matemática en la complejidad en sentipensar decolonial. Rev.Int. de Form.de Professores (RIFP), Itapetininga, v. 5, e020012, p. 1-23, 2020c.

RODRÍGUEZ, M. Mesetas biográficas-narrativas de la enseñanza de la matemática. Rev.Int. de Pesq. em Didática das Ciências e Matemática (RevIn), Itapetininga, v. 1, e020001, p. 1-21, 2020d.

SKOVSMOSE, O. Investigación, práctica, incertidumbre y responsabilidad. En Valero, P. y Ole Skovsmose, O. Educación Matemática crítica. Una visión sociopolítica del aprendizaje y la enseñanza de las matemáticas (p. 261-370). Colombia: Universidad de los Andes, Centro de Investigación y Formación en Educación, 2012.

Recebido em: 21 de maio de 2020

Aceito em: 17 de setembro de 2020 